\title{
Midterm Outcome of Total Knee Arthroplasty in East Coast Population of Hospital Tengku Ampuan Afzan Kuantan
}

\author{
Mohd Shahidan Noor Rahin ${ }^{1}$, Ahmad Hafiz Dzulkifly ${ }^{1}$, Harris Anwar Ali ${ }^{2}$ \\ ${ }^{1}$ Department of Orthopaedics, Traumatology and Rehabilitation, Kulliyyah of \\ Medicine, International Islamic University Malaysia \\ ${ }^{2}$ Department of Orthopaedics, Hospital Taiping
}

Presenter: Mohd Shahidan Noor Rahin

Introduction: Total knee replacement (TKR) is an excellent advancement in medical treatment which gives good joint function and pain relief for degenerative diseases of joint since it was introduced in Malaysia since 1970. The purpose of this study was to evaluate the midterm functional outcome of patients undergone TKR using the NexGen LPS Flex prosthesis between January 2005 until April 2006 in primary osteoarthritis patients in our local east coast population. Materials and Methods: Forty six patients, $87 \%$ female $(n=40)$ and $13 \%$ male $(n=6)$, aged 54 to 81 (mean, 69 \pm 5.69 ) years underwent primary TKR using the hi-flex knee prosthesis (Zimmer) performed by two different surgeons were followed up for a minimal of five years. All used the same technique of surgery, medial parapatellar incision, cruciate substitute and cemented. Assesment were made based on the quality of life outcome score (SF-36) and the Oxford-12 knee scoring system questionnaires. Results: Oxford -12 revealed that patients had mild problems in terms of function and pain with the average function and pain score of $14.35 \pm 4.21$ and $9.28 \pm 3.01$ compared to a maximum possible score of 35 and 25 respectively. For eight health domains of SF-36, patients had the highest score (i.e. good health) for role emotional (RE), followed by mental health $(\mathrm{MH})$, and social functioning (SF). This resulted in a higher score for mental component summary (MCS) than physical component summary (PCS). Since a low score of Oxford-12 indicates a better state of health compared to high score for SF-36, negative correlation between them was expected. Conclusion: The NexGen LPS-Flex TKR had good midterm functional outcome. Overall, TKR were found to be effective in terms of improvement in health-related quality-of-life dimensions. 